Table 1. MRI-detected enthesitis versus clinical examination

\begin{tabular}{lccr}
\hline & \multicolumn{3}{c}{ Plantar fascia MRI } \\
\hline Clinical examination for enthesitis & No enthesitis $(\mathrm{n}=74)$ & Enthesitis $(\mathrm{n}=5)$ & K-value \\
\hline Normal, no (\%) & $72(97)$ & $3(60)$ & 0.41 \\
Clinically suspect, no (\%) & $2(3)$ & $2(40)$ & \\
& \multicolumn{2}{c}{ Achilles tendon MRI } & \\
Clinical examination for enthesitis & No enthesitis $(\mathrm{n}=70)$ & Enthesitis $(\mathrm{n}=9)$ & $\mathrm{k}$-value \\
Normal, no (\%) & $68(97)$ & $8(89)$ & 0.12 \\
Clinically suspect, no (\%) & $2(3)$ & $1(11)$ & \\
\hline * & &
\end{tabular}

${ }^{*}=$ This investigator-initiated study was funded by Janssen Inc.
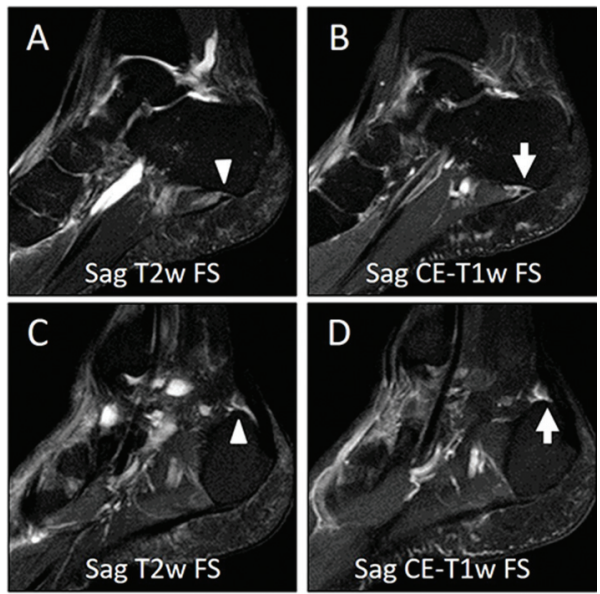

Figure 1. Plantar fascia $(A \& B)$ and Achilles tendon $(C \& D)$ with increased T2 signal reflecting edema (arrowhead) and contrast enhancement (arrow) around the entheses.

Disclosure of Interests: Nienke J. Kleinrensink: None declared, Emmerik Leijten: None declared, Wouter Foppen Grant/research support from: WF has received research grants from NovoNordisk and Pfizer. All were paid to the institution.

Paid instructor for: WF has received paid instructor fees from Pfizer. All were paid to the institution., Suzanne C. Diepstraten: None declared, Nanette L. Vincken: None declared, Pim de Jong: None declared, Timothy R. Radstake: None declared

DOI: 10.1136/annrheumdis-2019-eular.2399

\section{SAT0533 MRI ASSESSMENT OF HIP JOINTS INVOLVEMENT IN EARLY AXIAL SPONDYLOARTHRITIS PATIENTS}

Ekaterina Agafonova, Tatiana Dubinina, Daria Rumiantceva, Anastasiya Demina, Shandor Erdes. Federal State Budgetary Scientific Institution "Research Institute of Rheumatology named after V.A.Nasonova, laboratory spondylarthritis, Moscow, Russian Federation

Background: Based on available data from epidemiological studies in Russia different degree of hip involvement was found in $46 \%$ of ankylosing spondylitis (AS) patients, but only $7 \%$ of all cases resulted in total hip replacement procedures.

Objectives: To compare the ultrasound (US) manifestations of hip arthritis (coxitis) with the results of magnetic resonance imaging (MRI) of the hip joints $(\mathrm{HJ})$ in patients with early axial spondyloarthritis (AxSpa).

Methods: We examined 39 patients (mean age $28 \pm 5.92$ years) with a diagnosis of AxSpa (ASAS criteria 2009), with prescription inflammatory back pain no more than 5 years; age $<45$ years with US of coxitis. The median duration of the disease was 21 [3-60] months. BASDAl $4.0 \pm$ 2.0, BASFI $2.0[0.4 ; 3.5]$. All patients underwent clinical, radiological, ultrasound, and MRI scans of $\mathrm{HJ}$. For ultrasound coxitis was considered an increase in cervical-capsular distance (CCD) more than $7 \mathrm{~mm}$. Coxitis is found in 27 men and 12 women. The CCD median was 7.5 [7.0; 8.2] $\mathrm{mm}$.

Results: All patients in addition to ultrasound signs of coxitis had MRI signs of inflammation of the HJ. Pain in the $\mathrm{HJ}$ was determined in 33 $(85 \%)$ patients. The relationship between the level of pain and the amount of effusion in $\mathrm{HJ}$ was not detected. RCC significantly correlated with laboratory parameters of disease activity, such as ESR $(r=0.34) p$ $<0.05$ and CRP $(r=0.3), p<0.05$. X-ray examination revealed radiological changes in 7 patients (BASRI hip II-III). The following inflammatory changes (IC) were identified in the study population using MRI: synovitis was established in 36 (92\%) patients, bone marrow edema (BME) - in 1 $(3 \%)$, and combination of synovitis with BME was observed in 2 patients (5\%)

Conclusion: Ultrasound allows you to detect changes in $\mathrm{H}$ in the early stages of the disease. It is possible to use ultrasound as a screening method to determine the presence of synovitis in the TBS. MRI HJ with AxSpa, allows to determine whether the patient has inflammatory changes, including in the absence of radiological changes in these joints. In $15 \%$ of cases, coxitis is asymptomatic at early stages, which requires a thorough examination of patients with AxSpa. In addition to clinical and radiological examination, all patients were subjected to MRI with T1 and STIR modes.

Disclosure of Interests: : Ekaterina Agafonova: None declared, Tatiana Dubinina: None declared, Daria Rumiantceva: None declared, Anastasiya Demina: None declared, Shandor Erdes Consultant for: Development of studies concepts., Speakers bureau: Educational meetings organized or supported by companies.

DOI: 10.1136/annrheumdis-2019-eular.3522

\section{SAT0534 MAGNETIC RESONANCE IMAGING MARKERS IMPROVE THE PREDICTION MODEL FOR TOTAL KNEE REPLACEMENT OVER 13 YEARS IN OLDER ADULTS}

Benny Antony ${ }^{1}$, Ishanka Munugoda ${ }^{1}$, Dawn Aitken ${ }^{1}$, Petr Otahal $^{1}$,

Michelle Lorimer' ${ }^{2}$, Stephen Graves ${ }^{3}$, Johanne Martel-Pelletier ${ }^{4}$, Jean-

Pierre Pelletier ${ }^{4}$, Flavia Cicuttini ${ }^{5}$, Graeme Jones ${ }^{1} .{ }^{1}$ University of Tasmania, Menzies Institute for Medical Research, Hobart, Australia; ${ }^{2}$ Australian Orthopaedic Association National Joint Replacement Registry (AOANJRR), Adelaide, Australia; ${ }^{3}$ South Australian Health and Medical Research Institute (SAHMRI), Adelaide, Australia; ${ }^{4}$ Osteoarthritis Research Unit, University of Montreal Hospital Research Centre, Montreal, Canada; ${ }^{5}$ Department of Epidemiology and Preventive Medicine, Monash University, Melbourne, Australia

Background: Quantitative and semi-quantitative measurement of structural changes on MRI have been widely adopted in knee osteoarthritis (OA) research. However, few long-term studies describe the independent association of these structural factors and total knee replacement (TKR) in older adults.

Objectives: To describe whether cartilage defects, bone marrow lesions (BMLs), effusion-synovitis, and meniscal pathologies at baseline are associated with TKR over 13 years and to estimate the additive effect of these measures for risk prediction of TKR.

Methods: 1082 participants (mean age 62.8 years, 50\% female) were randomly recruited from Tasmania and followed over 13.3 years. A 1.5T MRI scan of the right knee was acquired at baseline $(n=930)$. Cartilage defects (grade 0-4), BMLs (grade 0-3), effusion-synovitis (grade 0-3), meniscal tears (grade 0-3) and meniscal extrusion (grade 0-2) was scored at baseline using T1-weighted and T2-weighted MRI. WOMAC knee pain was recorded from questionnaires and radiographic OA (ROA) was assessed by OARSI scale. The incidence of primary (first-time) TKR was determined by data linkage to the Australian Orthopaedic Association National Joint Replacement Registry (AOANJRR). Log binomial regression were used to estimate the risk of TKR associated with baseline MRI measures, adjusting for age, sex, BMI, ROA, and pain. Receiver operating characteristic $(\mathrm{ROC})$ analyses were used and area under the curves (AUCs) were compared.

Results: After adjustment for age, sex, BMI, and ROA status, baseline cartilage defects (grade 2, $R R=6.71 ;$ grade $3, R R=11.17$; grade 4 , $R R=13.2$; all $p<0.01$ ) were consistently and significantly associated with TKR over 13 years. These associations remained significant after further adjustment for other MRI pathologies. BMLs (grade 1, RR=2.74; grade 2, $R R=2.64$; grade $3 R R=4.01$, all $p<0.05$ except grade 2 ), grade 3 effusion-synovitis $(R R=2.74, p<0.05)$ and suprapatellar effusion-synovitis area were associated with TKR over 13 years. However, these associations of BMLs and effusion-synovitis with TKR were largely dependent on cartilage defects. Those who had TKR all had a meniscal tear at baseline, with $96 \%$ of them having a grade 3 tear.

Compared to the baseline model with age, sex and BMI (area under the ROC curve [AUC $=0.62]$ ), Model 1 with the addition of ROA and WOMAC pain performed better $(A \cup C=0.77$ ). Addition of cartilage defects to Model 1 resulted in a significant increase of AUC to 0.84. However, addition of BMLs and effusion-synovitis to the model 1 resulted in a nonsignificant small increase in AUC (0.78 and 0.76 ) only. The combination of all MRI pathologies to Model 1 resulted in a significant increase of AUC to 0.82 .

Conclusion: Baseline knee MRI structural pathology markers can predict TKR over the long-term, suggesting that MRI structural markers are good predictors of rapid knee OA progression in the general population. 